\title{
Effect of mass transfer on the free convective flow through a porous medium with variable permeability in slip flow regime with couple stresses
}

\author{
${ }^{1}$ Nidhish Kumar Mishra, ${ }^{2}$ Vineet Kumar Sharma and ${ }^{1}$ Debangana Rajput \\ 1 Department of Mathematics, D. S. College, Aligarh (U. P.) \\ ${ }^{2}$ Deptt. of Mathematics, Rawal Institute of Engineering and Technology, Faridabad \\ E-mail: vineetmathematics@gmail.com
}

\begin{abstract}
This paper examines the problem of mass transfer on MHD unsteady free convective flow of a polar fluid through a porous medium of variable permeability bounded by an infinite horizontal porous plate in slip flow regime. The permeability of the porous medium decreases exponentially with time about a constant mean. Using perturbation technique the expressions for the velocity distribution, mean angular velocity of rotation of particles, concentration distribution and skin friction are obtained. The effects of permeability parameter $\mathrm{K}_{0}$, Magnetic parameter $\mathrm{M}$, PrandtI number $P_{r}$, Schimdt number $S_{C}$, and Grashof number $G_{r}$, Modified Grashof number $G_{m}$ entering into the problem on velocity, temperature distribution and concentration distribution are shown graphically and discussed numerically. It can be observed that this velocity decreases with the increase in $M, P_{r}, S_{c}$ and increases with the increase in $K_{0}, G_{r}, G_{m}$. Temperature and Concentration decreases with the increase in the value of $P_{r}$ and $S_{c}$ respectively.
\end{abstract}

\section{INTRODUCTION}

The convection problem in a porous medium has important application in geothermal reservoirs and geothermal energy extractions. It is obvious that in order to utilize the geothermal energy to maximum, one should have a complete and precise knowledge about the amount of perturbations needed to generate convection current in geothermal fluids. Also the knowledge of the quantity of perturbation is essential to entreat convection current in mineral fluids that is found in the earth's crust helps one to utilize minimal energy to extract the minerals. Cheng and Lau[1] and Cheng and Teckchandani [2] obtained numerical solutions for the convective flow in a porous medium bounded by two isothermal parallel plates in the presence of withdrawl of the fluid. All the above mentioned studies treat the permeability and the conductivity or thermal resistance of the medium as constant or neglect the effect of porosity. Lai and Kulacki [6] studied Coupled heat and mass transfer by natural convection from vertical surfaces in porous media. Lin and $\mathrm{Wu}[7]$ discussed Coupled heat and mass transfer by laminar natural convection from vertical plate. Malasetty and Gaikwad[8] investigated effect of cross diffusion on double diffusion convection in the presence of horizontal gradients. Yan et. al [11] have discussed Simultaneous heat and mass transfer in laminar mixed convection flows between vertical parallel plate with asymmetric heating. Yan[12] studied Turbulent mixed convection heat and mass transfer past a vertical porous plate. Mazumdar and Deka[9] investigated MHD flow past an impulsively started infinite vertical plate in presence of thermal radiation.

In geothermal region situation may arise when the flow becomes unsteady and sliping at the boundary may take place as well. In such situation of slip flow, ordinary continuum approach fails to yield satisfactory results. Many authors have solved problems taking slip conditions at the boundary. Jain and Teneja [3] solved the problem of magnetopolar flow through a porous medium in slip flow regime. Khandelwal et al [5] have studied effect of couple stresses on the flow through a porous medium with variable permeability in slip flow regime. Varshney et. al.[10] have discussed effect of heat transfer on the flow through a porous medium with variable permeability in slip flow regime with couple stress.

Our problem under study is an extension of the problem of Varshney et. al.[10] with mass transfer. The effects of different parameter entering into the problem viz. Permeability parameter $\mathrm{K}_{\mathrm{O}}$, Magnetic 
parameter $\mathrm{M}$, Prandtl number $\mathrm{P}_{\mathrm{r}}$, Schimdt number $S_{C}$, and Grashof number $G_{r}$, Modified Grashof number $G_{m}$ entering into the problem on velocity, temperature distribution and concentration distribution are shown graphically and discussed numerically

\section{FORMULATION AND SOLUTION OF THE PROBLEM}

Let us consider the unsteady flow of a polar fluid through a porous medium of variable permeability in slip flow regime past an infinite horizontal porous plate in presence of a transverse magnetic field. The external velocity of the fluid is assumed as $\mathrm{U}_{0}\left[1+\varepsilon \mathrm{e}^{-\mathrm{nt}}\right]$. The flow is chosen to be at small magnetic Reynolds number which enables us to neglect the induced magnetic field. The $x$-axis is taken along the plate and $y$-axis normal to it. Under these conditions the equations which govern the flow are:

$$
\begin{aligned}
\frac{\partial \mathrm{v}}{\partial \mathrm{y}}=0 & \\
\frac{\partial \mathrm{u}}{\partial \mathrm{t}}+\mathrm{v} \frac{\partial \mathrm{u}}{\partial \mathrm{y}}= & -\frac{1}{\rho} \frac{\partial \mathrm{p}}{\partial \mathrm{x}}+\left(v+v_{\mathrm{r}}\right) \frac{\partial^{2} \mathrm{u}}{\partial \mathrm{y}^{2}}+2 v_{\mathrm{r}} \frac{\partial \omega}{\partial \mathrm{y}} \\
& +g \beta\left(\mathrm{T}-\mathrm{T}_{\infty}\right)+g \beta^{\prime}\left(\mathrm{C}-\mathrm{C}_{\infty}\right) \\
& -\frac{\sigma \mathrm{B}_{0}^{2}}{\rho} \mathrm{u}-\frac{v}{\mathrm{~K}(\mathrm{t})} \mathrm{u}
\end{aligned}
$$

$\frac{\partial \omega}{\partial \mathrm{t}}+\mathrm{v} \frac{\partial \omega}{\partial \mathrm{y}}=\frac{\gamma}{\mathrm{I}} \frac{\partial^{2} \omega}{\partial \mathrm{y}^{2}}$

$\frac{\partial \mathrm{T}}{\partial \mathrm{t}}+\mathrm{v} \frac{\partial \mathrm{T}}{\partial \mathrm{y}}=\frac{\lambda}{\rho \mathrm{C}_{\mathrm{p}}} \frac{\partial^{2} \mathrm{~T}}{\partial \mathrm{y}^{2}}$

$\frac{\partial \mathrm{C}}{\partial \mathrm{t}}+\mathrm{v} \frac{\partial \mathrm{C}}{\partial \mathrm{y}}=\mathrm{D} \frac{\partial^{2} \mathrm{C}}{\partial \mathrm{y}^{2}}$

Where $\mathrm{T}$ and $\mathrm{C}$ are dimensional temperature and concentration, $u$ and $v$ are velocity in the direction of $x$ and $y, t$ is time, $g$ is acceleration due to gravity, $\beta$ and $\beta^{\prime}$ are coefficient of volume expansion due to temperature and concentration, $v_{\mathrm{r}}$ is the kinematic rotational viscosity, $\omega$ the mean angular velocity of rotation of the particles, $\mathrm{K}(\mathrm{t})$ the permeability of the porous medium, $\sigma$ the electrical conductivity, $\rho$ is density, $C_{p}$ is specific heat at constant pressure, $\lambda$ is thermal conductivity, $D$ is molar diffusivity, $\varepsilon$ is small reference parameter $<<1, B_{0}$ the magnetic induction, I a scalar constant of dimension equal to that of the moment of inertia of unit mass and

$$
\gamma=\frac{\mathrm{C}_{\mathrm{a}}+\mathrm{C}_{\mathrm{b}}}{\mathrm{I}}
$$

where $C_{a}$ and $C_{d}$ are coefficients of couple stress viscosities. Remaining symbols have their usual meaning.

The boundary conditions relevant to the problem are taken as

$$
\left.\begin{array}{c}
\mathrm{u}=\mathrm{L}_{1} \frac{\partial \mathrm{u}}{\partial \mathrm{y}}, \frac{\partial \omega}{\partial \mathrm{y}}=-\frac{\partial^{2} \mathrm{u}}{\partial \mathrm{y}^{2}}, \mathrm{~T}=\mathrm{T}_{\mathrm{w}}, \\
\mathrm{C}=\mathrm{C}_{\mathrm{w}} \text { at } \mathrm{y}=0 \\
\mathrm{u}=\mathrm{U}(\mathrm{t})=\mathrm{U}_{0}\left[1+\varepsilon \mathrm{e}^{-\mathrm{nt}}\right], \omega \rightarrow 0, \\
\mathrm{~T} \rightarrow \infty, \mathrm{C} \rightarrow \infty \text { at } \mathrm{y} \rightarrow \infty
\end{array}\right]
$$

Where $L_{1}=\left[\frac{2-m_{1}}{m_{1}}\right] L, L$ is the mean free path and $m_{1}$ is the Maxwell's reflection coefficient.

These boundary conditions are derived from the assumptions that the couple stresses are dominant during the rotation at the particles.

$$
\mathrm{K}(\mathrm{t})=\mathrm{K}_{0}\left[1+\varepsilon \mathrm{e}^{-\mathrm{nt}}\right]
$$

where $\mathrm{K}_{0}$ is the mean permeability of the medium, $A$ and $n$ are real positive constants, $t$ is the time and $\varepsilon$ is small such that $\varepsilon A<<1$.

Integration of equation (1) for constant suction gives,

$$
\mathrm{v}=-\mathrm{v}_{0}
$$

We introduce the following non - dimensional quantities : 
$\mathrm{u}^{*}=\frac{\mathrm{u}}{\mathrm{U}_{0}}, \quad \mathrm{y}^{*}=\frac{\mathrm{yv}_{0}}{\mathrm{v}}, \quad \mathrm{t}^{*}=\frac{\mathrm{t}^{2} \mathrm{v}_{0}}{\mathrm{v}}, \quad \mathrm{n}^{*}=\frac{\mathrm{vn}}{\mathrm{v}_{0}^{2}}$,

$\alpha=\frac{v_{\mathrm{r}}}{v}, \quad \beta=\frac{\mathrm{I} v}{\gamma}, \quad \theta=\frac{\mathrm{T}-\mathrm{T}_{\infty}}{\mathrm{T}_{\mathrm{w}}-\mathrm{T}_{\infty}}, \quad \phi=\frac{\mathrm{C}-\mathrm{C}_{\infty}}{\mathrm{C}_{\mathrm{w}}-\mathrm{C}_{\infty}}$

$\mathrm{G}_{\mathrm{r}}=\frac{v g \beta}{\mathrm{U}_{0} \mathrm{v}_{0}^{2}}\left(\mathrm{~T}_{\mathrm{w}}-\mathrm{T}_{\infty}\right) \quad$ (Grashof Number),

$\mathrm{G}_{\mathrm{m}}=\frac{v g \beta}{\mathrm{U}_{0} \mathrm{v}_{0}^{2}}\left(\mathrm{C}_{\mathrm{w}}-\mathrm{C}_{\infty}\right)$ (Modified Grashof Number)

$\mathrm{M}^{2}=\frac{\sigma \mathrm{B}_{0}^{2} v}{\rho \mathrm{v}_{\mathrm{o}}^{2}}$ (Hartman Number),

$\mathrm{P}_{\mathrm{r}}=\frac{v \rho \mathrm{C}_{\mathrm{p}}}{\lambda}$ (Prandtl Number),

$\mathrm{h}_{1}=\frac{\mathrm{L}_{1} \mathrm{~V}_{0}}{\mathrm{v}}$ (Rarefaction Parameter $)$

$\omega^{*}=\frac{\nu \omega}{\mathrm{v}_{0} \mathrm{U}_{0}}$ (Non-Dimensional Amplitude Ratio),

$\mathrm{K}_{0}^{*}=\frac{\mathrm{v}_{0}^{2} \mathrm{~K}_{0}}{v^{2}}$ (Permeability Parameter)

$\mathrm{S}_{\mathrm{c}}=\frac{\mathrm{v}}{\mathrm{D}}$ (Schmidt Number $)$

In view of equations (6) and (7) and for the free stream velocity, the equations of motion in nondimensional form after dropping the asterisks over them reduce to:

$$
\begin{aligned}
& (1+\alpha) \frac{\partial^{2} \mathrm{u}}{\partial \mathrm{y}^{2}}+\frac{\partial \mathrm{u}}{\partial \mathrm{y}}+2 \alpha \frac{\partial \omega}{\partial \mathrm{y}} \\
& -\left[\mathrm{M}^{2}+\frac{1}{\mathrm{~K}_{0}\left(1+\mathrm{A} \varepsilon \mathrm{e}^{-\mathrm{nt}}\right)}\right](\mathrm{u}-\mathrm{U})-\frac{\partial \mathrm{u}}{\partial \mathrm{t}} \\
& =-\frac{\partial \mathrm{u}}{\partial \mathrm{t}}-\mathrm{G}_{\mathrm{r}} \theta-\mathrm{G}_{\mathrm{m}} \phi
\end{aligned}
$$

$\frac{\partial^{2} \omega}{\partial \mathrm{y}^{2}}+\beta \frac{\partial \omega}{\partial \mathrm{y}}=\beta \frac{\partial \omega}{\partial \mathrm{t}}$

$\frac{\partial^{2} \theta}{\partial \mathrm{y}^{2}}+\mathrm{P}_{\mathrm{r}} \frac{\partial \theta}{\partial \mathrm{y}}=\mathrm{P}_{\mathrm{r}} \frac{\partial \theta}{\partial \mathrm{t}}$

$\frac{\partial^{2} \phi}{\partial \mathrm{y}^{2}}+\mathrm{S}_{\mathrm{c}} \frac{\partial \phi}{\partial \mathrm{y}}=\mathrm{S}_{\mathrm{c}} \frac{\partial \phi}{\partial \mathrm{t}}$

with corresponding boundary conditions

$$
\left.\begin{array}{c}
\mathrm{u}=\mathrm{h}_{1} \frac{\partial \mathrm{u}}{\partial \mathrm{y}}, \frac{\partial \omega}{\partial \mathrm{y}}=-\frac{\partial^{2} \mathrm{u}}{\partial \mathrm{y}^{2}}, \theta=1, \\
\phi=1 \quad \text { at } \mathrm{y}=0 \\
\mathrm{u} \rightarrow \mathrm{U}(\mathrm{t})=\left[1+\varepsilon \mathrm{e}^{\mathrm{nt}}\right], \omega \rightarrow 0, \\
\theta \rightarrow 0, \phi \rightarrow 0 \quad \text { at } \mathrm{y} \rightarrow \infty
\end{array}\right]
$$

Since $\varepsilon$ is small reference parameter $\ll<1$, so let us assume $\mathrm{u}, \omega, \theta$ and $\phi$ as

$$
\left.\begin{array}{l}
\mathrm{u}(\mathrm{y}, \mathrm{t})=\mathrm{u}_{0}(\mathrm{y})+\varepsilon \mathrm{e}^{-\mathrm{nt}} \mathrm{u}_{1}(\mathrm{y}) \\
\omega(\mathrm{y}, \mathrm{t})=\omega_{0}(\mathrm{y})+\varepsilon \mathrm{e}^{-\mathrm{nt}} \omega_{1}(\mathrm{y}) \\
\theta(\mathrm{y}, \mathrm{t})=\theta_{0}(\mathrm{y})+\varepsilon \mathrm{e}^{-\mathrm{nt}} \theta_{1}(\mathrm{y}) \\
\phi(\mathrm{y}, \mathrm{t})=\phi_{0}(\mathrm{y})+\varepsilon \mathrm{e}^{-\mathrm{nt}} \phi_{1}(\mathrm{y})
\end{array}\right]
$$

We get the solution after neglecting the coefficients of $\varepsilon^{2}$ and using corresponding boundary conditions:

$$
\begin{aligned}
u= & \left(\begin{array}{l}
1+C_{3} e^{-R_{2} y}+Z_{1} C_{1} e^{-\beta y} \\
-Z_{2} e^{-P_{r} y}-Z_{3} e^{-S_{c} y}
\end{array}\right) \\
& +\varepsilon e^{-n t}\left(\begin{array}{l}
1+C_{4} e^{-R_{4} y}-Z_{9} e^{-R_{2} y} \\
-Z_{10} e^{-\beta y}+Z_{11} e^{-P_{r} y} \\
+Z_{12} e^{-S_{c} y}+C_{2} Z_{13} e^{-m_{1} y}-\frac{M^{2} A}{N^{2}-n}
\end{array}\right)
\end{aligned}
$$

$\omega=\mathrm{C}_{1} \mathrm{e}^{-\beta \mathrm{y}}+\varepsilon \mathrm{e}^{-\mathrm{nt}} \mathrm{C}_{2} \mathrm{e}^{-\mathrm{m}_{1} \mathrm{y}}$ 


$$
\begin{aligned}
& \theta=\mathrm{e}^{-\mathrm{P}_{\mathrm{r}} \mathrm{y}} \\
& \phi=\mathrm{e}^{-\mathrm{S}_{\mathrm{c}} \mathrm{y}}
\end{aligned}
$$

Where $\mathrm{R}_{1}=\frac{-1+\sqrt{1+4(1+\alpha) \mathrm{N}^{2}}}{2(1+\alpha)}$,

$$
\begin{aligned}
& \mathrm{R}_{2}=\frac{1+\sqrt{1+4(1+\alpha) \mathrm{N}^{2}}}{2(1+\alpha)} \\
& \mathrm{R}_{3}=\frac{-1+\sqrt{1+4(1+\alpha)\left(\mathrm{N}^{2}-\mathrm{n}\right)}}{2(1+\alpha)}, \\
& \mathrm{R}_{3}=\frac{1+\sqrt{1+4(1+\alpha)\left(\mathrm{N}^{2}-\mathrm{n}\right)}}{2(1+\alpha)} \\
& \mathrm{m}_{1}=\frac{\beta+\sqrt{\beta^{2}-4 \mathrm{n} \beta}}{2}, \\
& \mathrm{~N}^{2}=\mathrm{M}^{2}+\frac{1}{\mathrm{~K}_{0}}
\end{aligned}
$$$$
\mathrm{C}_{1}=\frac{\mathrm{R}_{2} \mathrm{C}_{3}\left(\mathrm{Z}_{1} \mathrm{Z}_{6}+\mathrm{Z}_{3} \mathrm{Z}_{7}\right)+\mathrm{Z}_{4}\left(\mathrm{Z}_{4} \mathrm{P}_{\mathrm{r}}^{2}+\mathrm{Z}_{3} \mathrm{~S}_{\mathrm{c}}^{2}\right)}{\left(\mathrm{Z}_{2} \mathrm{Z}_{8}-\mathrm{R}_{2}^{2} \mathrm{Z}_{1} \mathrm{Z}_{5}\right)}
$$$$
\mathrm{C}_{2}=\frac{\left(1+\mathrm{R}_{4} \mathrm{~h}_{1}\right) \mathrm{Z}_{15}-\mathrm{R}_{4}^{2} \mathrm{Z}_{14}}{\mathrm{Z}_{13}\left(1+\mathrm{h}_{1} \mathrm{~m}_{1}\right) \mathrm{R}_{4}^{2}-\left(\mathrm{Z}_{13} \mathrm{~m}_{1}^{2}+1\right)\left(1+\mathrm{R}_{4} \mathrm{~h}_{1}\right)}
$$$$
\mathrm{C}_{3}=\frac{\mathrm{Z}_{1} \mathrm{Z}_{5}\left(\mathrm{Z}_{4} \mathrm{P}_{\mathrm{r}}^{2}+\mathrm{Z}_{3} \mathrm{~S}_{\mathrm{c}}^{2}\right)-\mathrm{Z}_{8}\left(\mathrm{Z}_{1} \mathrm{Z}_{6}+\mathrm{Z}_{3} \mathrm{Z}_{7}\right)}{\left(\mathrm{Z}_{2} \mathrm{Z}_{8}-\mathrm{R}_{2}^{2} \mathrm{Z}_{1} \mathrm{Z}_{5}\right)}
$$$$
\mathrm{C}_{4}=\frac{\left(\mathrm{Z}_{13} \mathrm{~m}_{1}^{2}-1\right) \mathrm{Z}_{14}-\mathrm{Z}_{13}\left(1+\mathrm{h}_{1} \mathrm{~m}_{1}\right) \mathrm{Z}_{15}}{\mathrm{Z}_{13}\left(1+\mathrm{h}_{1} \mathrm{~m}_{1}\right) \mathrm{R}_{4}^{2}-\left(\mathrm{Z}_{13} \mathrm{~m}_{1}^{2}+1\right)\left(1+\mathrm{R}_{4} \mathrm{~h}_{1}\right)}
$$

$$
\begin{aligned}
& \mathrm{Z}_{1}=\frac{2 \alpha \beta}{(1+\alpha) \beta^{2}-\beta-\mathrm{N}^{2}}, \\
& \mathrm{Z}_{2}=\frac{\mathrm{G}_{\mathrm{r}}}{(1+\alpha) \mathrm{P}_{\mathrm{r}}^{2}-\mathrm{P}_{\mathrm{r}}-\mathrm{N}^{2}}, \\
& \mathrm{Z}_{3}=\frac{\mathrm{G}_{\mathrm{m}}}{(1+\alpha) \mathrm{S}_{\mathrm{c}}^{2}-\mathrm{S}_{\mathrm{c}}-\mathrm{N}^{2}} \mathrm{Z}_{4}=1+\mathrm{h}_{1} \mathrm{R}_{2}, \\
& \mathrm{Z}_{5}=1+\mathrm{h}_{1} \beta, \mathrm{Z}_{6}=1+\mathrm{h}_{1} \mathrm{P}_{\mathrm{r}}, \mathrm{Z}_{7}=1+\mathrm{h}_{1} \mathrm{~S}_{\mathrm{c}} \text {, } \\
& \mathrm{Z}_{8}=1+\mathrm{Z}_{1} \beta^{2} \\
& \mathrm{Z}_{9}=\frac{(1+\alpha) \mathrm{AR}_{2}^{2} \mathrm{C}_{3}+\left(\mathrm{M}^{2}-1\right) \mathrm{AR}_{2} \mathrm{C}_{3}}{(1+\alpha) \mathrm{R}_{2}^{2}-\mathrm{R}_{2}-\left(\mathrm{N}^{2}-\mathrm{n}\right)}, \\
& \mathrm{Z}_{10}=\frac{(1+\alpha) \mathrm{AZ}_{1} \mathrm{C}_{1} \beta^{2}+\left(\mathrm{M}^{2}-1\right) \mathrm{AZ}_{1} \mathrm{C}_{1} \beta-2 \alpha \mathrm{A} \beta \mathrm{C}_{1}}{(1+\alpha) \beta^{2}-\beta-\left(\mathrm{N}^{2}-\mathrm{n}\right)} \\
& \mathrm{Z}_{11}=\frac{(1+\alpha) \mathrm{AZ}_{2} \mathrm{P}_{\mathrm{r}}^{2}+\left(\mathrm{M}^{2}-1\right) \mathrm{AZ}_{2} \mathrm{P}_{\mathrm{r}}-\mathrm{AG}_{\mathrm{r}}}{(1+\alpha) \mathrm{P}_{\mathrm{r}}^{2}-\mathrm{P}_{\mathrm{r}}-\left(\mathrm{N}^{2}-\mathrm{n}\right)}, \\
& \mathrm{Z}_{12}=\frac{(1+\alpha) \mathrm{AZ}_{3} \mathrm{~S}_{\mathrm{c}}^{2}-\left(\mathrm{M}^{2}-1\right) \mathrm{AZ}_{3} \mathrm{~S}_{\mathrm{c}}-\mathrm{AG}_{\mathrm{m}}}{(1+\alpha) \mathrm{S}_{\mathrm{c}}^{2}-\mathrm{S}_{\mathrm{c}}-\left(\mathrm{N}^{2}-\mathrm{n}\right)} \\
& \mathrm{Z}_{13}=\frac{2 \alpha \mathrm{m}_{1}}{(1+\alpha) \mathrm{m}_{1}^{2}-\mathrm{m}_{1}-\left(\mathrm{N}^{2}-\mathrm{n}\right)} \\
& \mathrm{Z}_{14}=\left(1+\mathrm{h}_{1} \mathrm{~S}_{\mathrm{c}}\right) \mathrm{Z}_{12}+\left(1+\mathrm{h}_{1} \mathrm{P}_{\mathrm{r}}\right) \mathrm{Z}_{11} \\
& -\left(1+\mathrm{h}_{1} \beta\right) \mathrm{Z}_{10}-\left(1+\mathrm{h}_{1} \mathrm{R}_{2}\right) \mathrm{Z}_{9} \\
& \mathrm{Z}_{15}=\mathrm{Z}_{12} \mathrm{~S}_{\mathrm{c}}^{2}+\mathrm{Z}_{11} \mathrm{P}_{\mathrm{r}}^{2}-\mathrm{Z}_{10} \beta^{2}-\mathrm{Z}_{9} \mathrm{R}_{2}^{2}
\end{aligned}
$$

The non-dimensional skin friction at the plate is given by

$$
\begin{aligned}
& \tau=\frac{\tau_{\mathrm{w}}}{\rho \mathrm{U}_{0} \mathrm{v}_{0}}=(1+\alpha)\left(\frac{\partial \mathrm{u}}{\partial \mathrm{y}}\right)_{\mathrm{y}=0} \\
& =(1+\alpha)\left(-\mathrm{R}_{2} \mathrm{C}_{3}-\mathrm{Z}_{1} \mathrm{C}_{1} \beta+\mathrm{Z}_{2} \mathrm{P}_{\mathrm{r}}+\mathrm{Z}_{3} \mathrm{~S}_{\mathrm{c}}\right)
\end{aligned}
$$




$$
+\varepsilon \mathrm{e}^{-\mathrm{nt}}(1+\alpha)\left(\begin{array}{l}
-\mathrm{C}_{4} \mathrm{R}_{4}-\mathrm{Z}_{9} \mathrm{R}_{2}+\mathrm{Z}_{10} \beta \\
-\mathrm{Z}_{11} \mathrm{P}_{\mathrm{r}}-\mathrm{Z}_{12} \mathrm{~S}_{\mathrm{c}}-\mathrm{C}_{2} \mathrm{Z}_{13} \mathrm{~m}_{1}
\end{array}\right)
$$

RESULTS AND DISCUSSION:
The zeroth-order velocity profile $\mathrm{u}_{0}$ is tabulated in Table-1and plotted in Fig.-1 having Graph-1 to 7 at $\alpha=0.2, \beta=2, h_{1}=0.4, \varepsilon=0.01, A=0.5$ and different values of $K_{0}, M, P_{r}, S_{C}, G_{r}$ and $G_{m}$.

\begin{tabular}{|c|c|c|c|c|c|c|}
\hline & K0 & M & Pr & Sc & Gr & Gm \\
\hline Graph 1 & 1 & 0.2 & 0.3 & 0.5 & 5 & 4 \\
\hline Graph 2 & 2 & 0.2 & 0.3 & 0.5 & 5 & 4 \\
\hline Graph 3 & 1 & 2.0 & 0.3 & 0.5 & 5 & 4 \\
\hline Graph 4 & 1 & 0.2 & 0.7 & 0.5 & 5 & 4 \\
\hline Graph 5 & 1 & 0.2 & 0.3 & 0.6 & 5 & 4 \\
\hline Graph 6 & 1 & 0.2 & 0.3 & 0.5 & 10 & 4 \\
\hline Graph 7 & 1 & 0.2 & 0.3 & 0.5 & 5 & 6 \\
\hline
\end{tabular}

From the Table- 1 and Graphs of Fig.- 1 it is clear that the zeroth-order velocity $\mathrm{u}_{0}$ continuously decreases on increasing $\mathrm{y}$. It can also be observed that this velocity decreases with the increase in $M, P_{r}, S_{c}$ and increases with the increase in $K_{0}, G_{r}, G_{m}$

Table - 1

\begin{tabular}{|c|c|c|c|c|c|c|c|}
\hline y & Graph-1 & Graph - 2 & Graph - 3 & Graph - 4 & Graph - 5 & Graph-6 & Graph-7 \\
\hline 0 & 8.8350 & 12.0600 & 8.5000 & 8.5400 & 8.3650 & 11.4950 & 10.1500 \\
\hline 1 & 6.2043 & 8.4169 & 4.5640 & 5.0847 & 5.1790 & 8.2925 & 6.8740 \\
\hline 2 & 4.5225 & 6.0472 & 2.9518 & 3.2389 & 3.5969 & 6.1219 & 4.8602 \\
\hline 3 & 3.4157 & 4.4712 & 2.1728 & 2.2390 & 2.7228 & 4.6279 & 3.5754 \\
\hline 4 & 2.6753 & 3.4098 & 1.7446 & 1.6917 & 2.1926 & 3.5882 & 2.7406 \\
\hline
\end{tabular}


Am. J. Sci. Ind. Res., 2011, 2(3): 469-477

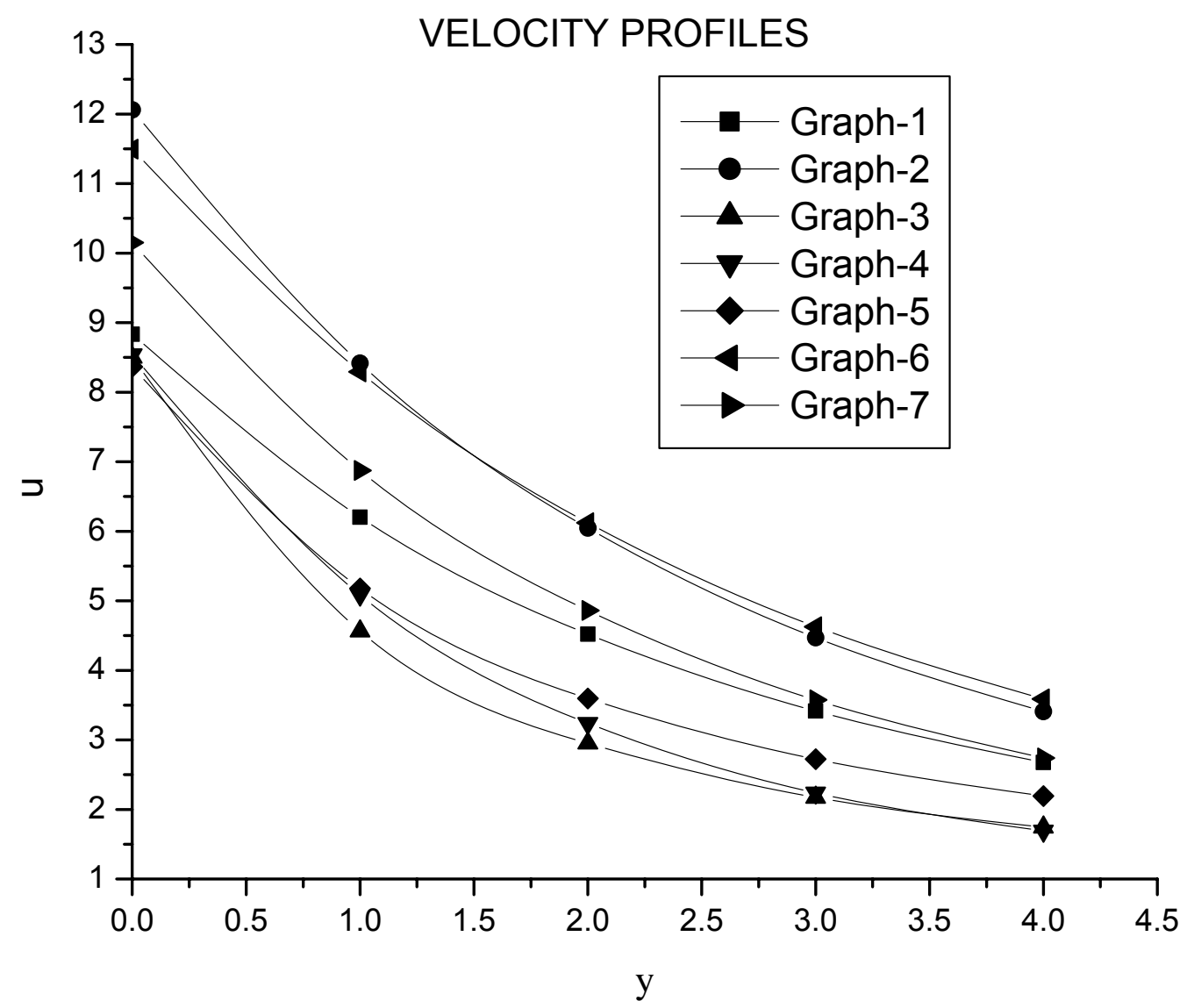

Figure-1

The Temperature profile is tabulated in table- 2 and plotted in Fig.-2. It is observed from figure -3 that all temperature graphs decrease with the increase in $y$. It is noticed that temperature decreases, when the value of Pr increases.
The concentration profile is tabulated in table- 3 and plotted in Fig.-3. It is observed from figure -3 that all concentration graphs decrease with the increase in $y$. It is also noticed that concentration decreases with the increase in value of Sc. 
Table - 2

\begin{tabular}{|c|c|c|c|}
\hline $\mathrm{y}$ & $\operatorname{Pr}=0.71$ & $\operatorname{Pr}=1.0$ & $\operatorname{Pr}=2.0$ \\
\hline 0 & 1 & 1 & 1 \\
\hline 1 & 0.491644 & 0.367879441 & 0.135335283 \\
\hline 2 & 0.241714 & 0.135335283 & 0.018315639 \\
\hline 3 & 0.118837 & 0.049787068 & 0.002478752 \\
\hline 4 & 0.058426 & 0.018315639 & 0.000335463 \\
\hline
\end{tabular}

TEMPERATURE PROFILES

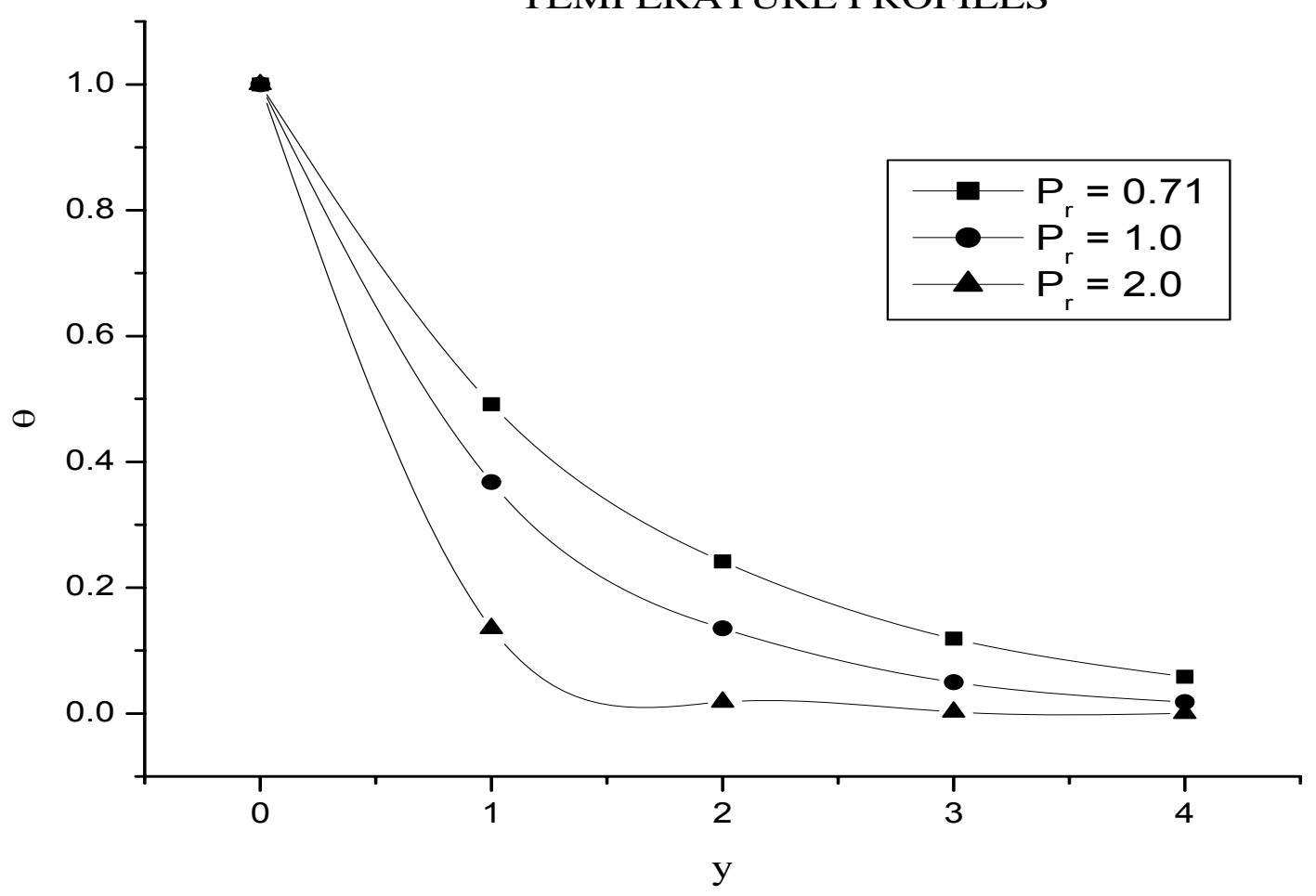

Figure-2 
Table - 3

\begin{tabular}{|c|c|c|c|}
\hline $\mathrm{y}$ & $\mathrm{Sc}=1.5$ & $\mathrm{Sc}=2.5$ & $\mathrm{Sc}=3.5$ \\
\hline 0 & 1 & 1 & 1 \\
\hline 1 & 0.22313 & 0.082084999 & 0.030197383 \\
\hline 2 & 0.049787 & 0.006737947 & 0.000911882 \\
\hline 3 & 0.011109 & 0.000553084 & $2.75364 \mathrm{E}-05$ \\
\hline 4 & 0.002479 & $4.53999 \mathrm{E}-05$ & $8.31529 \mathrm{E}-07$ \\
\hline
\end{tabular}

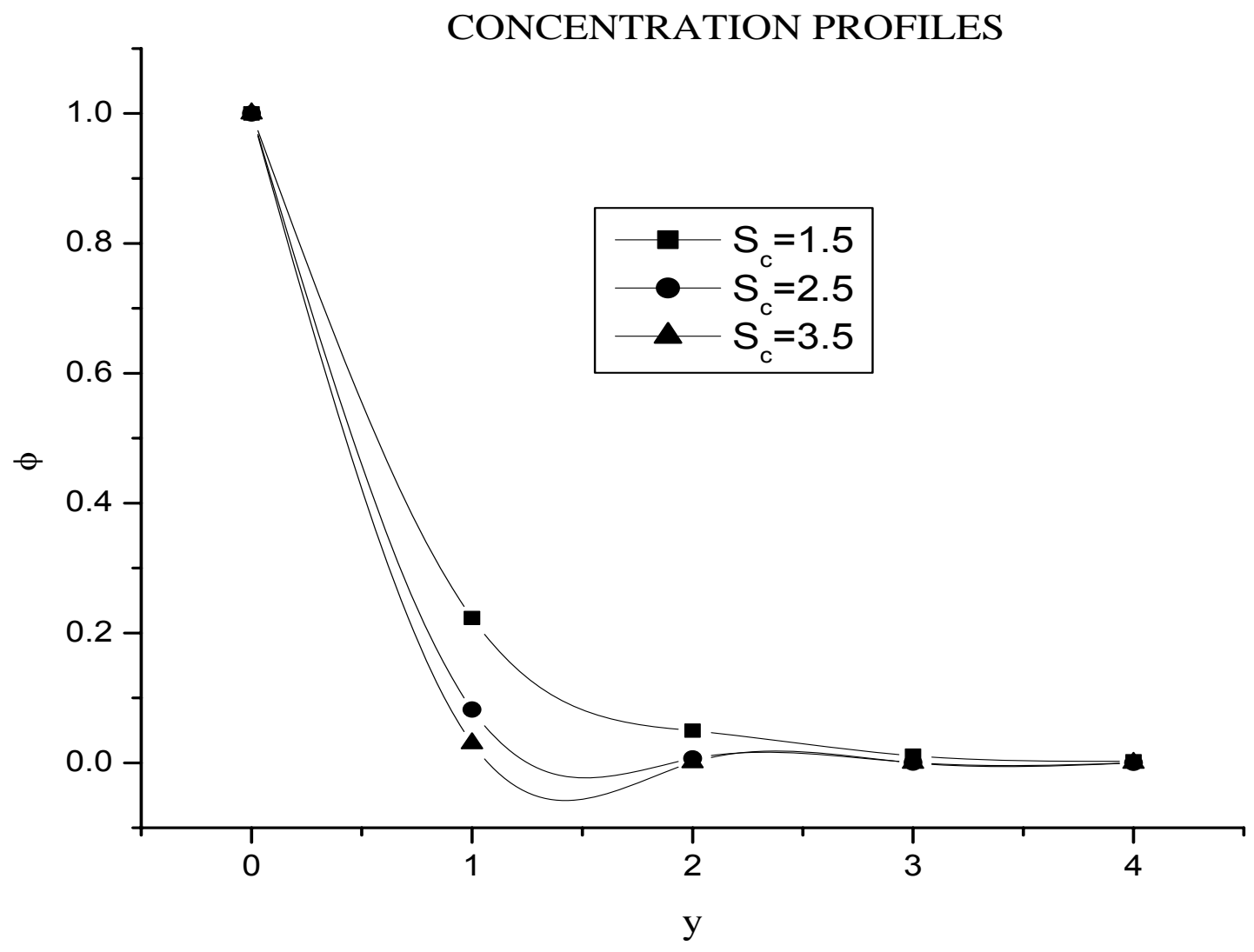

Figure-3 
Am. J. Sci. Ind. Res., 2011, 2(3): 469-477

\section{REFERENCES:}

[1] Cheng, P. and Lau, K.H.: In Proc., $2^{\text {nd }}$ National Symp. Development, Geothermal Resources, 1591- 1598 (1977).

[2] Cheng, P.,I and Teckchandani, L.: In Symposium National Phys. Prop. Earth's crust Vail Colobola, 2-6. August (1976).

[3] Jain, N.C. and Taneja, Rajeev: Problem of magnetopolar flow through a porous medium in slip flow regime, Ganita Sandesh, 14, 87 (2000).

[4] Kaviany, M., Principles of Heat Transfer in Porous Media, MacGraw-Hill, New York, (1993).

[5] Khandelwal, K. Anil; Gupta, Poonam and Jain, N.C.: Ganita, Effect of couple stresses on the flow through a porous medium with variable permeability in slip flow regime Vol. 54 , No. 2, 203-212 (2003).

[6] Lai, F.C and Kulacki, F.A.: Coupled heat and mass transfer by natural convection from vertical surfaces in porous media, Int. J. Heat Mass Transfer, 24, 1189-1194 (1991).

[7] Lin, H.T and Wu, C.M.: Coupled heat and mass transfer by laminar natural convection from vertical plate, Int. J. Heat Mass Transfer, 30, 369376 (1995).

[8] Malasetty, M.D and Gaikwad, S.N.: Effect of cross diffusion on double diffusion convection in the presence of horizontal gradients, Int. J. Engg., 40, 773-789 (2002).

[9] Mazumdar, M.K. and Deka, R.K.: MHD flow past an impulsively started infinite vertical plate in presence of thermal radiation, Romanian Journal of Physics, 52(5-7), 565-573 (2007).

[10] Varshney, N.K; Kumari, S. and Sharma, Vineet Kumar.: Effect of hest transfer on the flow through a porous medium with variable permeability in slip flow regime with couple stress, Jour. PAS, Vol. 15 (Mathematical Science), pp71-79, (2009).

[11] Yan, W.N; Tsay, Y.L. and Lin, T.I.: Simultaneous heat and mass transfer in laminar mixed convection flows between vertical parallel plate with asymmetric heating, Int. J. Heat fluid flow, 10, 262-269 (1989).

[12] Yan, W.N: Turbulent mixed convection heat and mass transfer past a vertical porous plate, ASME, J. Heat Transfer, 117 229-233 (1995). 